\title{
http://dx.doi.org/10.35381/r.k.v4i1.461
}

\section{El talento humano, factor clave para la gestión organizacional en Instituciones de intermediación financiera}

\section{Human talent, a key factor for organizational management in financial intermediation institutions}

\author{
Pedro Eloy Lema Guiracocha \\ plema@psg.ucacue.edu.ec \\ Universidad Católica de Cuenca \\ Ecuador \\ https://orcid.org/0000-0001-6900-0998 \\ Juan Carlos Erazo Álvarez \\ jcerazo@ucacue.edu.ec \\ Universidad Católica de Cuenca \\ Ecuador \\ https://orcid.org/0000-0001-6480-2270 \\ Cecilia Ivonne Narváez Zurita \\ inarvaez@ucacue.edu.ec \\ Universidad Católica de Cuenca \\ Ecuador \\ https://orcid.org/0000-0002-7437-9880
}

Recibido: 10 de agosto de 2019

Aprobado: 30 de agosto de 2019

\section{RESUMEN}

La gestión del talento se basa en la legitimización de que la potencialidad humana se puede relacionar con los procesos productivos eficientes, eficaces y efectivos con el fin de mejorar la calidad del servicio. La unidad de análisis de la presente investigación es la Cooperativa de Ahorro y Crédito de la pequeña empresa Biblián Ltda., en la que se pudo evidenciar un deficiente desempeño laboral traducido en un nivel de servicio inadecuado e insatisfacción de los socios. En función a este planteamiento se propone desarrollar un modelo de gestión del talento humano que incorpore aspectos fundamentales de la organización. La metodología utilizada fue de tipo no experimental, descriptiva y transversal. Los resultados más relevantes evidenciaron falencias en la 
comunicación, el desempeño, cumplimiento de objetivos y la falta de actualización de procesos.

Descriptores: Gestión; Talento Humano; Mejora del Servicio, Desempeño, Servicios Financieros.

\begin{abstract}
Talent management is based on the legitimization that human potentiality can be related to efficient, effective and effective production processes in order to improve service quality. The unit of analysis of the present investigation is the Cooperativa de Ahorro y Crédito de la pequeña empresa Biblián Ltda., In which it was possible to demonstrate a poor work performance translated into an inadequate service level and dissatisfaction of the members. Based on this approach, it is proposed to develop a human talent management model that incorporates fundamental aspects of the organization. The methodology used was non-experimental, descriptive and transversal approach. The most relevant results showed flaws in communication, performance, fulfillment of objectives and lack of updating processes.
\end{abstract}

Key words: Management; Human Resources; Service Improvement; Performance; Financial Services.

\title{
INTRODUCCIÓN
}

Según el artículo 311, Según el artículo 311, de la constitución de la república del Ecuador, el sector de la economía popular y solidaria está conformada por cooperativas, bancos y cajas comunales, cajas de ahorro y entidades asociativas, las cuales están llamadas a ser parte del desarrollo productivo de la economía de los pueblos, entendiéndose por economía popular y solidaria a un sector que se diferencia por la utilización de su propia fuerza de trabajo, además por ofrecer servicios financieros a través del crédito, ahorro, y servicios complementarios, los mismos que se encuentran sustentados en procesos, tecnología, talento humano, acorde a las necesidades de los pueblos, contribuyendo de esta el desarrollo socioeconómico de un país. Este sector pretende integrar a todos los actores de la población a través de un trato justo, incluyente y solidario bajo una verdadera convicción de inclusión financiera.

En los últimos siete años, las cooperativas de ahorro y crédito (COAC) dieron un salto importante. Mientras en el 2012 sus activos sumaban USD 6, 027 millones, al cierre del 
año pasado (2018) sumaron 14, 016 millones, según la Superintendencia de la Economía Popular y Solidaria (SEPS). Ese mismo comportamiento también se observa en variables como los pasivos, el patrimonio, los créditos, los depósitos, las utilidades y el número de socios.

Para la Superintendencia de Economía Popular y Solidaria, este dinamismo refleja la confianza de los clientes y es resultado del control que tienen las instituciones. La Superintendencia fue creada en el 2012 y entre sus competencias está la regulación de un sector que está en proceso de consolidación.

Actualmente las cooperativas de ahorro y crédito mantienen el $26 \%$ de participación del sistema financiero, según datos publicados a abril por la superintendencia de economía popular y solidaria, esto se debe a la confianza de los socios hacia las cooperativas, además por que las cooperativas han logrado penetrar con la inclusión financiera en zonas rurales en donde el índice de pobreza es mayor, sector en donde la banca privada ha descuidado a ese segmento de clientes. Además, Juan Pablo Guerra añade que el sector cooperativo ha creado un modelo dinámico en la economía del país con la participación de estos actores como son las Coac's.

Es así que las instituciones financieras como las cooperativas de ahorro y crédito al mantener este ritmo acelerado de crecimiento y al brindar servicios financieros obliga a mejorar la propuesta de valor hacia los socios/clientes, usuarios, brindando un servicio de calidad hacia los socios, entonces esto hace que las organizaciones deben mantener y fortalecer su capital humano, ya que una empresa es tan buena como lo sean sus empleados es así que, a mayor capacidad del equipo humano, las, las actividades, procesos y tareas que se realicen dentro de la organización serán más eficientes lo que les permitirá cumplir con sus objetivos estratégicos.

En la actualidad para que una organización se mantenga en el tiempo y cumpla sus objetivos estratégicos, no debe dejar de lado los recursos financieros, tecnológicos que se complementan con el talento humano, por esta razón la diferencia entre una y otra organización es la forma en la que comprenden al talento humano, así como el rol que le atribuyen dentro de la empresa. 
La entidad, objeto de investigación es la cooperativa de ahorro y crédito de la pequeña empresa Biblián Ltda. La misma que presenta la siguiente información financiera, así como datos generales, los mismos que son los siguientes: La cooperativa a julio de 2019 cuenta con 14 sucursales distribuidas en las provincias del Azuay y Cañar, con un total de 255.501.816,34 millones en activos, un pasivo de 215.212.785,57 millones y un patrimonio de 37.715.047,93, además mantiene alrededor de 80,000 socios, 180 empleados, su oficina matriz está ubicada en el Cantón Biblián Provincia del Cañar.

La cooperativa entre sus objetivos estratégicos es el de fortalecer su capital humano, a través de una evaluación de desempeño y el de mejorar la comunicación interna, los mismos que han presentado un leve deterioro, en este sentido la cooperativa no podrá ser competitiva ni cumplir con sus objetivos si no se realiza un proceso adecuado de selección, contratación, capacitación y evaluación de personal, motivo por el cual la presente investigación tiene como objetivo analizar los aspectos más relevantes que posibiliten potenciar la gestión del talento humano de la cooperativa de ahorro y crédito de la pequeña empresa Biblián Ltda., a través de la formulación de estrategias, así como el de fortalecer las políticas institucionales que permita mejorar la productividad y desempeño de los empleados.

\section{Un modelo de gestión de talento humano hacia los nuevos escenarios laborales}

Actualmente las nuevas organizaciones requieren de personal con nuevas habilidades, competencias, trabajo en equipo, es decir verdaderos líderes que coadyuven al desarrollo de las actividades y metas de cada uno de los empleados, esta evolución se debe también al desarrollo tecnológico en cada una de las organizaciones, razón por la cual un modelo de gestión de talento humano debe estar enfocado a las nuevas tendencias del mercado laboral, como a los diferentes cambios normativos, los mismos que me permitirán alcanzar los objetivos estratégicos, crear una cultura organizacional sólida, así como el ser más competitivos y sostenibles en el tiempo. (Cordero, Narváez y Erazo, 2019) 
Tejada (2003) manifiesta que "la gestión del talento se basa en la legitimización de que la potencialidad humana se puede relacionar con los procesos productivos eficientes, eficaces y efectivos...", mientras que el papel de la gerencia sería "...que adquiera también conocimientos que le permitan detectar, apoyar, impulsar, poner a prueba e incentivar el talento de sus colaboradores y el suyo propio; todo esto como una nueva alternativa de cambio organizacional".

La gestión de talento humano es un proceso en el cual requiere reclutar, seleccionar, recompensar, desarrollar y dar seguimiento a las personas, la cual me permitirá una toma de decisiones acertada, además es importante que las personas se sientan comprometidas con la empresa, lo cual ayudará a mejorar la productividad, calidad y cumplimiento de los objetivos, además me permitirá la optimización de tiempos y procesos. Entonces las organizaciones necesitan aprender a:

- Comprender la misión y visión de la empresa

- Romper paradigmas

- Innovar

- Enfrentarse al futuro

Temor a producir lo desconocido Yadira (2017) indica que la gestión del talento humano existe porque las organizaciones o empresas están conformadas por personas, ya que las organizaciones no podrían crecer ni sostenerse en el tiempo sin las personas, a través de una planificación adecuada de las actividades y planes de acción, por lo que las personas dependen de las organizaciones donde trabajan y lo realizan con el único propósito de alcanzar sus objetivos personales, desarrollo profesional y otras metas individuales.

Las personas dependen de las organizaciones para su crecimiento profesional, personal e individual, pero también las organizaciones dependen de las personas directamente ya sea para operar, producir bienes o servicios, atender a los clientes, competir en los mercados, cumplir con sus objetivos estratégicos, es por esta razón que existe una relación de mutua dependencia que proporciona beneficios recíprocos, es decir una simbiosis entra las personas y las organizaciones (Chiavenato, 2008). 
Dessler (2009) afirma que la gestión de talento humano son prácticas y políticas necesarias para manejar los asuntos que tienen que ver con las relaciones de trabajo, específicamente el de reclutar, evaluar, capacitar, remunerar y ofrecer un ambiente seguro y equitativo para los empleados" por lo que aseguramos contar con un personal idóneo en todas las áreas, el cual nos permitirá optimizar procesos, tiempo y recursos para las organizaciones.

Debido a la importancia que hoy en día se da a los trabajadores en las organizaciones el termino capital humano ha desaparecido, ya que actualmente es considerado como una persona dotada de habilidades y conocimientos que se han convertido en un aporte para el desarrollo, competencias, la productividad en las organizaciones (Ramos, 2014).

Alles (2009) Manifiesta que muchas organizaciones invierten en diferentes modelos de gestión de talento humano, pero luego no saben cómo conseguirlo y llevarlo a cabo, lo que busca el modelo es el de desarrollar las competencias de las personas basadas en tres subsistemas como, la selección que me permitirá contar con personal idóneo para cada área o función que desempeñen, evaluación del desempeño, este nos permitirá conocer el grado de las competencias con las que cuenten cada integrante de la organización y el ultimo y primordial es el desarrollo de las mismas. Borrero, (2019), añade la importancia de evaluar para adoptar la mejora continua en la organziación.

Actualmente las organizaciones tienen la ardua tarea de cubrir la brecha que existe entre las necesidades laborales actuales versus las exigencias del nuevo ecosistema laboral, la misma que debe empezar por romper ese viejo sistema jerárquico vertical y centrarnos en un nuevo modelo de gestión, decisión, trabajo en equipo con el propósito de crear una base para una cultura organizacional perdurable en el tiempo.

En resumen, la gestión de talento humano es crear un sistema entre lo social y lo económico es decir considerar al empleado como un factor motivacional con una concepción renovada, dinámica y competitiva, el mismo que me permita mejorar el desempeño y el clima laboral de las organizaciones.

El desempeño laboral podemos considerarlo como algo intrínseco de cada persona o individuo, es decir las cualidades y habilidades que interactúan entre sí, pero para que 
sean desarrolladas debe de existir una persona o responsable que identifique esas características para a través de ellas establecer estrategias que me permitirán cumplir con mis objetivos institucionales. (Cerón, Erazo,Ormaza y Narváez, 2019)

Robbins (1998) considera que los principales factores organizacionales que influyen en el desempeño laboral son:

- La capacidad del trabajador

* El cliente

* Actitud del trabajador

* Actitud percibida por el cliente

* Quejas y reclamos de los clientes

Para que exista un desempeño laboral en una organización, también debe existir una motivación hacia el personal, ya que al no existir un personal que se encuentre motivado muy difícilmente una organización alcanzara sus objetivos estratégicos, tampoco podrá generar una cultura organizacional que pueda mantenerse en el tiempo.

Una cualidad o condición para que la gestión de talento humano se relacione con los procesos de manera eficiente, eficaz y efectiva dependerá de la fortaleza de su talento humano con la que cuente cada una de las organizaciones, pero también esto se complementa con que la gerencia o las personas responsables, también adquieran nuevos conocimientos que les permitan detectar, apoyar, impulsar, e incentivar el talento de sus colaboradores, todo esto con un solo propósito como es el cambio organizacional. Para alcanzar el éxito en una organización, el personal debe de encontrarse motivado y comprometido con la institución, además de encontrarse en permanente capacitación, para tener la suficiente capacidad de identificar, captar las oportunidades de mejora en cada uno de los procesos, pero siempre preguntando a los demás colaboradores sobre la manera que se está gestionando, si es la adecuada o no, para de esta forma seguir retroalimentándonos y mejorando cada día más en cada una de sus actividades y tareas. Al considerarse un área muy sensible dentro de las organizaciones, también es importante considerar una serie de variables como, la cultura, estructura, las características, es decir los procesos internos de una organización y todo aquello que se 
encuentre vinculado con el talento humano dentro de una organización (Chiavenato, 2008).

Existen modelos de gestión de talento humano, cada uno diferentes entre sí, pero todos con un solo objetivo que es el de mantener niveles de eficiencia, estos modelos de gestión empresarial se van integrando y esto hace que cada día vayan evolucionando hacia nuevos modelos con el propósito de mejorar su competitividad y sus resultados empresariales trazados.

Un proceso moderno de gestión de talento humano se basa en una admisión de personas, seguida con el reclutamiento y selección, evaluación, compensación y un monitoreo continúo basados en sistemas de información, innovación y una base de datos actualizada, así como a las necesidades de la organización, permitiendo de esta forma contar con personal adecuado e idóneo en cada una de las áreas.

Además, se indica que el talento humano es considerado como un proceso administrativo, es decir que se debe planear, organizar, dirigir y controlar al talento humano de una organización permitiendo de esta forma contar con las habilidades y conocimientos del sujeto de una organización (Rodríguez, 2013).

El talento humano también es considerado como una herramienta estratégica, ya que a través de ello se puede impulsar las competencias individuales y colectivas de una organización alcanzando un nivel de excelencia, el mismo que me permitirá enfrentar a nuevos desafíos en el medio (Mora, 2012).

\section{El desempeño laboral como un factor esencial para el logro de objetivos en una organización.}

Un factor que incide en el desempeño laboral es el clima laboral de una organización, ya que de ello dependerá el éxito o fracaso de una empresa, al no tener a un empleado que se sienta parte de la institución en donde exista una buena comunicación, definido de manera adecuada los procesos, tener una estructura adecuada en lo referente al orgánico funcional en donde las persona tenga claro cuáles son sus deberes y obligaciones. 
También el no proporcionar retroalimentación oportuna incide en el desempeño, es así que cuando solo se mide y no se retroalimenta de manera adecuada y permanente, mantendremos el mismo nivel siempre, solo con la ilusión de que las cosas se están haciendo bien, cayendo en una zona de confort, creando resistencia al cambio en las organizaciones, por lo que es importante que las organizaciones realicen un seguimiento luego de los resultados de la evaluación, brindando retroalimentación oportuna y adecuada en cada una de las áreas de la organización.

En consecuencia, es trascendental fortalecer el capital humano de las organizaciones, el cual me permitirá elevar sus conocimientos, y habilidades para brindar una atención personalizada a los socios/clientes, además fortaleceríamos la cultura organizacional, y por ende se cumplirían los objetivos institucionales planteados en el corto y largo plazo. El desempeño laboral es considerado como acciones o comportamientos de los empleados considerados como de mayor importancia en una empresa, los mismos que podrían ser medidos a través de las competencias individuales para poder identificar su nivel de aportación hacia la organización (García, 2001).

Los diferentes cambios que van teniendo las organizaciones, hace que se dé la importancia al área de talento humano, para que sea esta la encargada de atraer retener, contratar al personal adecuado para la empresa, ya que el reto de las organizaciones es de evolucionar e innovar las herramientas, procesos y técnicas relacionadas al desarrollo organizacional y las necesidades del mercado laboral.

El talento humano en las organizaciones hoy en día están llamadas a generar y contribuir ventajas competitivas que me permitan cumplir con los objetivos, así como con la visión y misión de la empresa, ya que esto hará que se diferencie con el resto de competidores, para lo cual se tiene que organizar los procesos de una manera adecuada y oportuna, estableciendo claramente cuáles son sus etapas, responsabilidades, recursos para su ejecución, pero siempre manteniendo esa relación entre el personal y la organización, ya que esto será un factor clave para mejorar el desempeño laboral en una empresa.

Además, consideramos también que para que exista un desempeño laboral adecuado, es importante contar con políticas claras y bien definidas, esto hará que todos los 
empleados al menos cumplan con sus procesos y tareas en los plazos y tiempos definidos en dicha política. Pero para que una política tenga éxito esta deberá ser monitoreada y controlada de manera permanente, también es importante la socialización y comunicación de la política ya que el éxito de una organización dependerá mucho de cómo y quién comunique. Para mantener un desempeño laboral efectivo, es necesario que las instituciones cuenten con un plan de incentivos para su personal. Aldana \& Piña (2017), señalan que esto además permite concebir un servicio de calidad al cliente.

También para que exista un desempeño laboral efectivo, es necesario considerar un factor importante como lo es la comunicación, ya que a través de la misma se puede realizar las actividades y tareas a través de procesos bien definidos y estructurados, optimizando de esta forma tiempo y recursos para la organización.

\section{METODOLOGÍA}

La presente investigación es de tipo no experimental ya que los datos fueron tomados a través de una encuesta a los empleados de la Cooperativa, tal como lo perciben o se comportan los fenómenos estudiados, para poder determinar el desempeño laboral de los empleados de la Cooperativa. Sin que exista una manipulación o control de parte del investigador.

El enfoque es mixto por lo que se partió de hechos cuantitativos como son el número de personas que laboran en la institución, el tiempo de trabajo, género, etc. Resultados en porcentajes que se obtuvieron a través de la encuesta aplicada al personal de la institución, la misma que fue complementada con una entrevista al Jefe de Talento Humano de la Cooperativa, para validar y complementar los resultados obtenidos.

Es descriptiva ya que fue realizada a los empleados que laboran en la Cooperativa. En cada una de sus sucursales, departamentos, en el cual se obtuvieron información de las variables y dimensiones a través de las diferentes preguntas en la encuesta.

Es transversal porque la información fue recopilada en un momento determinado, a una población muestral que en este caso fue a 135 empleados de la Cooperativa. 
Para la presente investigación, se empleó, el método inductivo-deductivo para la formulación del problema de investigación.

Además, se empleó el método histórico-lógico para realizar las revisiones de la literatura que permitieron el desarrollo del marco teórico y del estado de la situación que sustentan a la investigación realizada.

Adicionalmente, también se empleó el método analítico-sintético que, en base al diseño y ejecución de encuestas y entrevistas, aporta al cumplimiento de los objetivos planteados en esta investigación.

\section{RESULTADOS}

En la investigación realizada se pudo identificar algunas debilidades que posee la cooperativa, sobre todo en algunos procesos dentro del área de talento humano.

En los resultados cuantitativos descritos en las siete variables analizadas: selección de personal, capacitación, compensación, evaluación, eficiencia, eficacia y comunicación, se ha considerado como de mayor relevancia las siguientes:

\section{Tabla 1.}

Resultados de la investigacion

\begin{tabular}{lcccc}
\multicolumn{1}{c}{ Variables } & Si & No & \multicolumn{2}{c}{ Porcentaje } \\
\hline Manual de procesos & 97 & 38 & $77 \%$ & $23 \%$ \\
Capacitación & 111 & 24 & $82 \%$ & $18 \%$ \\
Plan de incentivos & 102 & 33 & $76 \%$ & $24 \%$ \\
Productividad & 119 & 16 & $88 \%$ & $12 \%$ \\
Comunicación & 59 & 59 & $50 \%$ & $50 \%$ \\
\hline
\end{tabular}

Fuente: Encuestas realizadas al TH de la Cooperativa 
Se evidencia que la cooperativa cuenta con un manual de procesos de selección y contratación de personal, pero como aspectos a mejorar es la actualización de los manuales y procesos los mismos que tiene que estar acorde a las nuevas exigencias del mercado laboral, así como a los objetivos estratégicos de la institución.

Además, también se observa que la cooperativa cuenta con un plan de capacitación, en la que consta que las capacitaciones están dirigidas a todo el personal, que deberán ser replicadas a sus colaboradores, pero lo que se pudo evidenciar como oportunidades de mejora podría ser que el departamento de talento humano difunda y elabore un informe en el cual conste los cambios o avances que se han dado luego de la capacitación.

También se puede evidenciar claramente que la cooperativa cuenta con un plan de incentivos, entonces como oportunidades de mejora, tendríamos que identificar qué tipos de incentivos entrega la cooperativa a los empleados, y que incentivos le gustaría al personal.

Asi también se observa que las tareas encomendadas a los funcionarios son ejecutadas en los tiempos establecidos lo cual es visto de manera positiva por parte de los empleados, pero como aspectos a mejorar podemos manifestar que se tendría que identificar los tiempos por áreas y procesos para una toma de decisiones oportuna y adecuada.

También podemos observar que la cooperativa presenta debilidades en la comunicación, por lo que se tendría que identificar las causas del porque esa comunicación no es la adecuada, ya que en una organización que presenta debilidades en la comunicación muy difícilmente podrá cumplir sus objetivos estratégicos. 


\section{Propuesta de Modelo Gestión en la Cooperativa de Ahorro y Crédito de la Pequeña Empresa Biblián Ltda.}

La estructuración de un modelo de gestión de talento humano tiene como finalidad de: optimizar recursos, mejorar la prestación de servicios, mejorar la cultura organizacional, el mejoramiento continuo, la satisfacción y desempeño laboral, cumplimiento de objetivos estratégicos. Visto desde esa perspectiva se debe enfocar que los productos y servicios que brinda la cooperativa, vayan orientados a satisfacer las necesidades de sus socios y usuarios, esto a su vez permitirá generar un valor agregado a la institución, a sus colaboradores y socios en general, logrando así consolidarse en el mercado y sostenibles en el tiempo.

De ahí que, con la información obtenida se propone un modelo que mejore la gestión del talento humano de la Cooperativa, que me permita mejorar el desempeño laboral, que ayude a mejorar los servicios brindados de manera oportuna, mejorar los procesos, incrementar la eficiencia y eficacia de los empleados de la cooperativa en las diferentes áreas, alcanzar niveles adecuados de productividad, para de esta forma alcanzar los objetivos estratégicos y cumplir con su misión y visión institucional. Para ello, tal como se puede visualizar en la figura 2, es necesario vincular articuladamente las diferentes etapas de la gestión de talento humano en torno a los resultados que se quiere alcanzar, que en este caso es el de mejorar desempeño laboral de todos sus colaboradores de la institución. 
Figura 1. Propuesta de modelo de gestión de talento humano

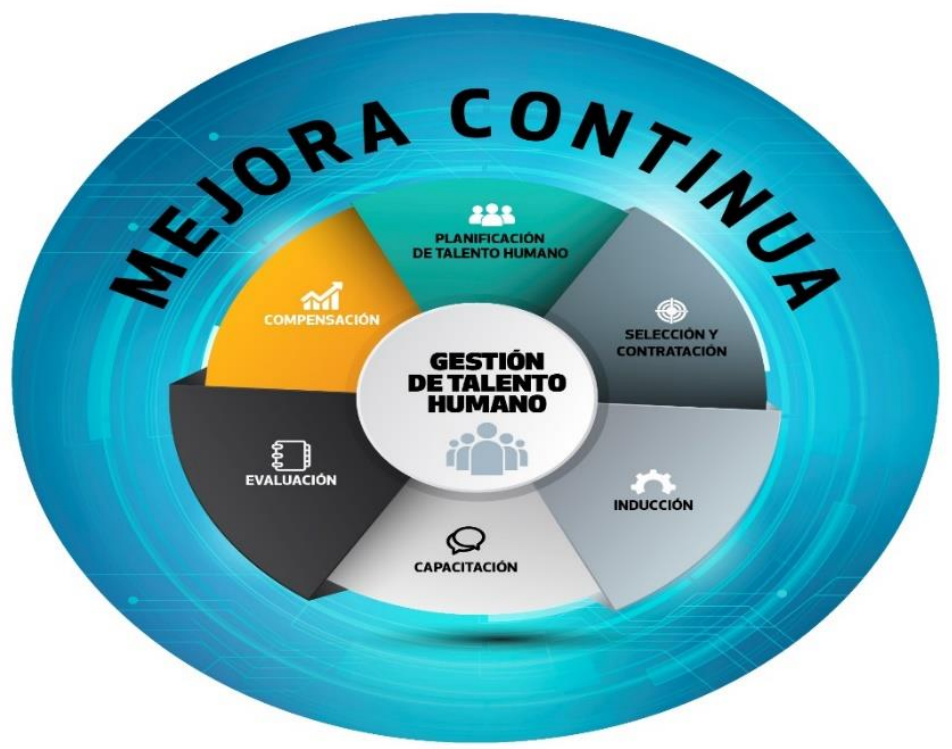

Fuente: Elaboración propia

El elemento dinamizador, del modelo propuesto, son los subsistemas, que conforman el sistema integrado del talento humano como es: la planificación del talento humano, la selección y contratación, la inducción, capacitación, evaluación y compensación, componentes que se encuentran debidamente relacionados con la misión y visión, objetivos estratégicos de la institución.

A continuación, realizaremos una breve descripción de los diferentes componentes que conforman el modelo de gestión de talento humano propuesto en la presente investigación para la cooperativa.

Para que un modelo de gestión de talento humano sea exitoso, debe empezar con una planificación de actividades, los mismos que deberán estar relacionados con los objetivos estratégicos de la institución.

Posteriormente se debe realizar un proceso de selección y contratación, bien estructurado porque de ello dependerá el éxito o fracaso de una organización, razón por 
la cual se propone que el proceso de selección y contratación de personal deberá contener lo siguiente,

Detección de necesidades: lo primero y fundamental que tenemos que tener en cuenta es conocer e identificar cuáles son las necesidades de la institución en toda su estructura organizacional

Definición del perfil del candidato: aquí debemos identificar las cualidades, habilidades que debe cumplir el candidato, es decir el nivel de conocimientos, experiencia, valores, niveles de estudio, idiomas, trabajo en equipo, trabajo bajo presión, etc.

Convocatoria: en esta etapa se recolectará el currículo de cada postulante, es decir los posibles aspirantes que cumplieron con los requisitos solicitados por la cooperativa, también es importante considerar los medios a través de la cual se va realizar la convocatoria, es decir, a través de las redes sociales, pagina web, prensa, etc.

Preselección: una vez que se ha recibido la información de los candidatos es importante realizar una primera selección, para de esta forma contar con los candidatos acorde a los requerimientos de la institución, con la cual se optimiza tiempo y recursos para la institución.

Selección: en este proceso existe diferentes maneras de selección, muchas organizaciones realizan entrevistas de trabajo, test psicotécnicos, pruebas profesionales, dinámicas de grupo, la utilización de una u otra técnica dependerá del puesto. Finalmente se realiza una entrevista a profundidad a los candidatos que hayan superado las distintas pruebas.

Informe de candidatos: aquí se recopila toda la información de los postulantes, en el cual deberá incluir los pro y contras de cada uno de los aspirantes, esta técnica me permitirá contar con una base de candidatos más idóneos, para los diferentes cargos o puestos a cubrir.

Toma de decisiones: en esta etapa es la más importante, ya que se va a elegir a la persona más idónea y cumpla con los perfiles que busca la institución.

Contratación: aquí es el momento en el que se firma el contrato, es decir esta etapa es de tipo legal básicamente 
Incorporación del puesto: es aquí en donde el candidato empieza a laborar en la organización, también algunas de las empresas en esta fase establecen periodos de prueba, aquí es la etapa en la que hay que presentar formalmente al nuevo empleado a todos sus colaboradores.

Formación: en esta etapa es en donde el área de talento humano o las áreas involucradas, procuren que el nuevo integrante se adapte lo más pronto posible a las tareas.

Seguimiento: Una última etapa y consideramos también importante como todas las demás, que para que un proceso de selección y contratación tenga éxito es necesario realizar una evaluación ya sea está a corto o largo plazo, la cual me permitirá identificar las falencias y ventajas para seguir mejorando en base a planes de acción propuestos por el responsable de talento humano.

A continuación, se ha realizado un flujograma del proceso de selección y contratación de personal.

Figura 2. Proceso de selección y contratación de personal

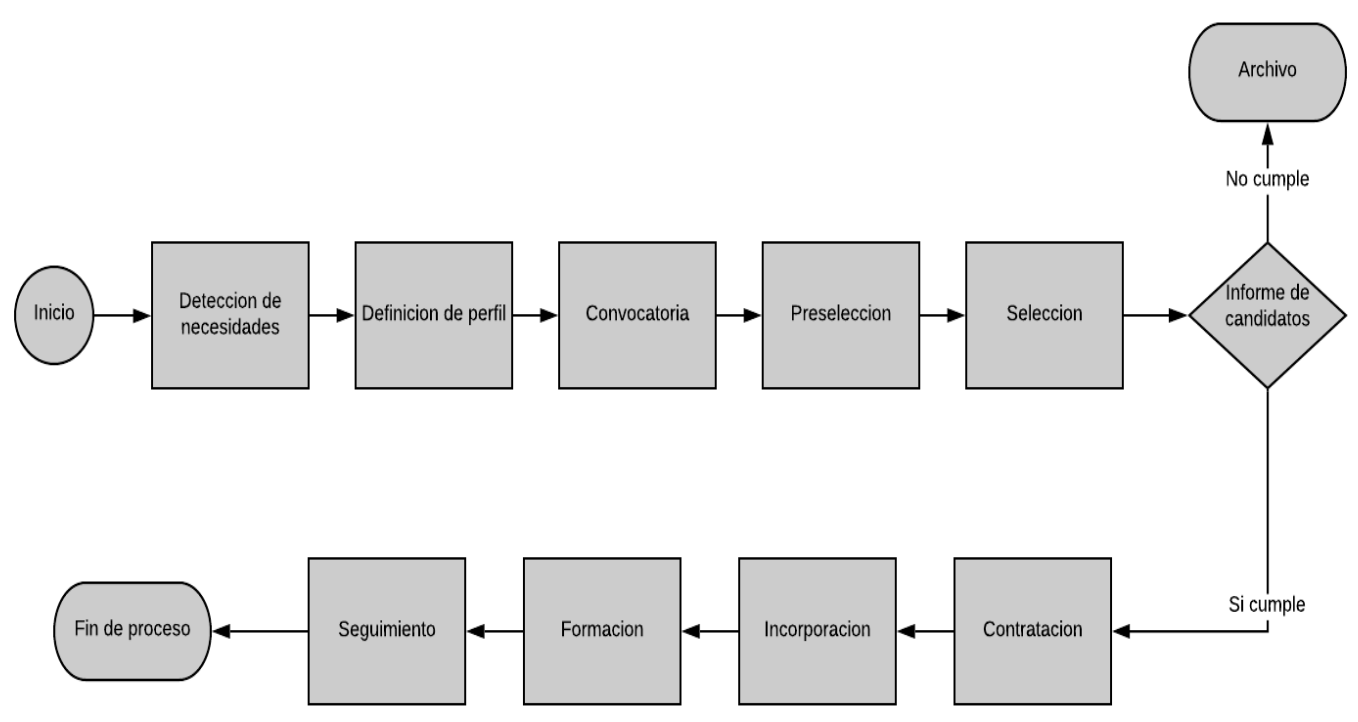

Fuente: Elaboración propia 
Todo este proceso debe estar ligado a las nuevas tendencias del mercado laboral, nuevas tecnologías, herramientas, que son claves para conseguir a los futuros empleados de la cooperativa, optimizando tiempo y recursos.

A través de este proceso de selección y contratación se busca contar con los mejores candidatos para cada puesto específico, ya que no es suficiente una persona con carrera y máster, sino también hacen falta de ciertas habilidades y destrezas personales para alcanzar los objetivos institucionales.

Además, consideramos también que es fundamental brindarle un proceso de inducción al personal, ya que a través de ello los nuevos empleados podrán contar con información respecto a los valores, antecedentes, objetivos estratégicos de la empresa, así como en cada uno de sus puestos de trabajo, muchas de las veces las empresas, suelen cometer el error de ignorar esta etapa, la misma que se ve reflejada en el corto plazo, cuando se realiza una evaluación.

Otra de las etapas que se debe tener en cuenta es la capacitación, aunque muchas de las organizaciones consideran a la capacitación como un gasto y no como una inversión que beneficiara tanto a la organización como a sus colaboradores.

Razón por la cual consideramos que en un modelo de gestión de talento humano no debe dejarse de lado a la capacitación ya que juega un papel primordial para el logro de los objetivos, tareas y proyectos, ya que es considerado como un proceso mediante el cual los trabajadores, adquieren los conocimientos, las herramientas, habilidades y actitudes para interactuar en el entorno laboral.

También podemos manifestar que a través de un programa de capacitación una organización se vería beneficiada en:

- Genera un aumento de la productividad y la calidad de trabajo.

- Incrementa la rentabilidad de la organización.

- Resolver los problemas generados el día a día.

- La supervisión.

- Estabilidad organizacional y flexibilidad. 
- Personal identificado con la empresa

A continuación, se plantea el siguiente plan de capacitación con el qué se pretende actualizar y ampliar los conocimientos de los empleados de la cooperativa, el mismo que me permitirá mantener un buen nivel de eficiencia y rendimiento colectivo, cumpliendo de esta forma con los objetivos estratégicos, optimizando procesos y recursos en la institución

A través de este plan de capacitación se pretende una alineación de su personal hacia los objetivos, su misión y visión, permitiendo además la solución a los problemas, preparándolos para enfrentar los cambios de su entorno. 


\section{Tabla 2}

\section{Plan de capacitación}

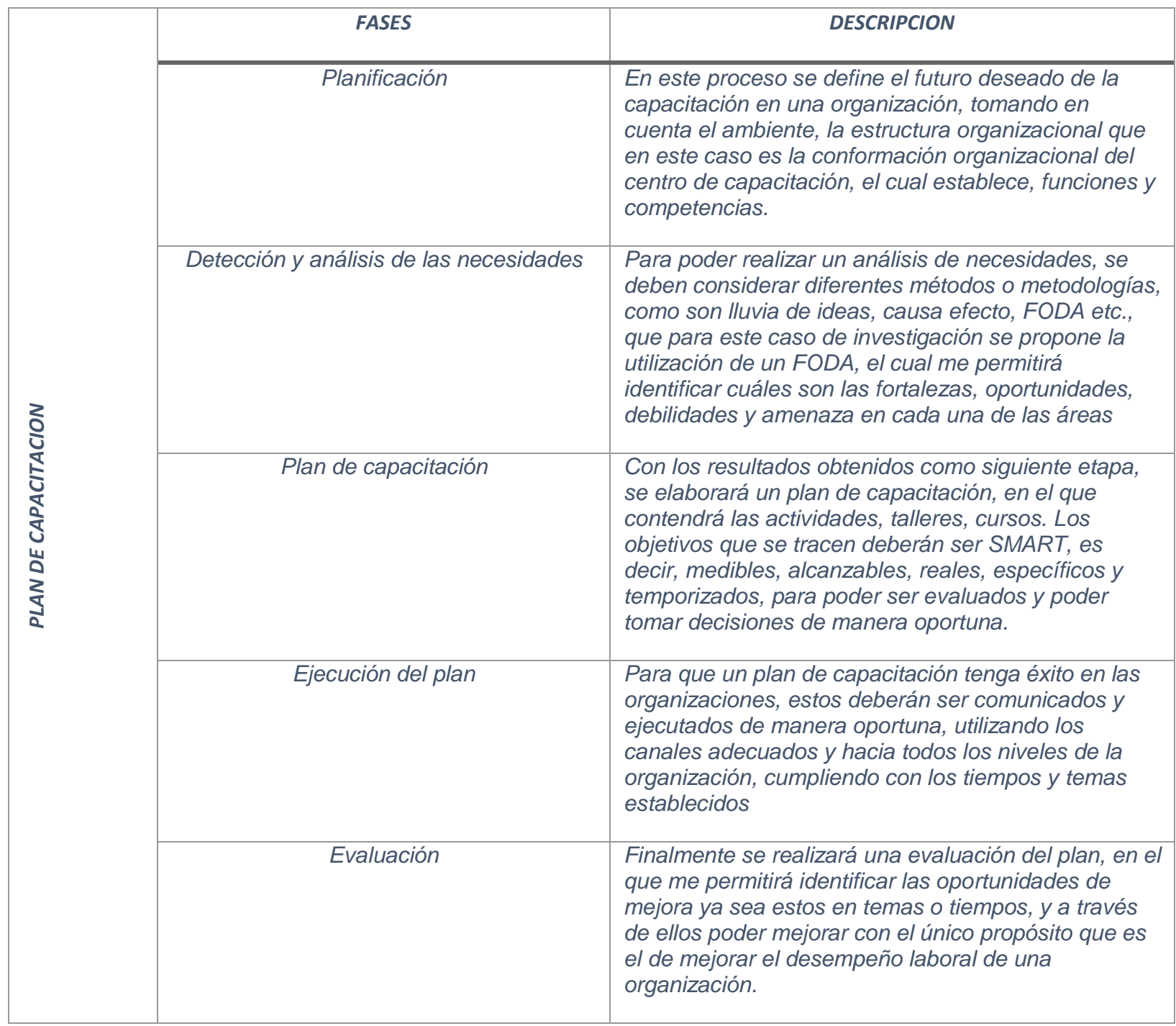

Fuente: Elaboración propia

También es importante considerar otro aspecto fundamental como es la evaluación al personal, porque a través de ello me permitirá conocer el grado de cumplimiento de los objetivos propuestos por la institución, su misión y visión de manera individual. Una organización al no realizar evaluaciones al personal, no podrá identificar las oportunidades de mejora, en sus procesos y procedimientos, así como sus debilidades. 
Para la realización de la evaluación se plantea realizar la evaluación 360 grados, ya que es una herramienta que me permite una evaluación integral, la cual es utilizada para medir las competencias de los colaboradores en una organización. Es una evaluación que se realiza al empleado, es decir lo realizan los compañeros, subordinados, supervisores, jefes directos, clientes internos y externos, basándose en el comportamiento y habilidades que tiene la persona en su entorno profesional.

La finalidad de la evaluación 360 grados, es el de brindar a cada uno de los colaboradores una retroalimentación para mejorar su desempeño y comportamiento de una manera más objetiva al contar con diferentes puntos de vista y perspectivas.

Aquí debemos tener en cuenta que la comunicación es una de las piezas claves para la correcta aplicación del modelo, por lo tanto, debe procurar a través de acciones concretas el fortalecimiento de las relaciones de confianza entre trabajadores.

Figura 3. Evaluación 360

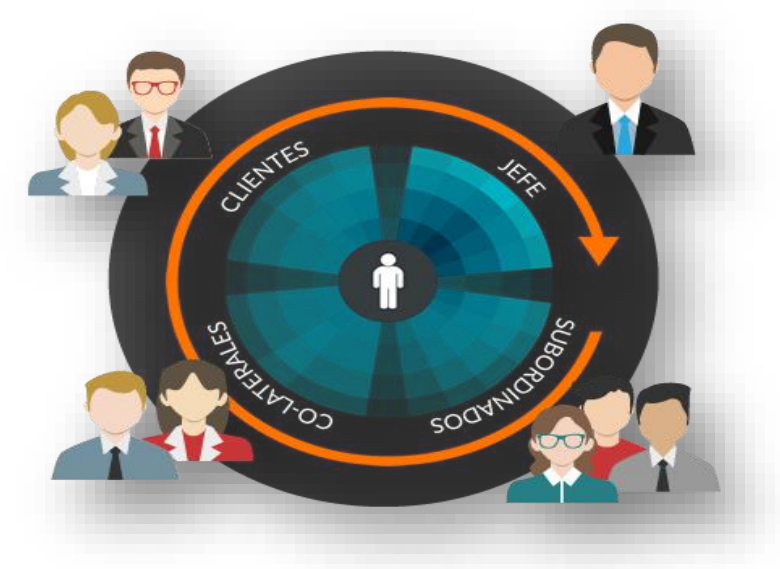

Fuente: Alles (2006)

Para la evaluación 360 grados consideramos que al menos se debe considerar los siguientes aspectos o factores que me permitirán obtener información, para una adecuada toma de decisiones, y al mismo tiempo me permita mejorar la productividad y 
el desempeño de los empleados de la cooperativa en todos sus niveles. A continuación, se presenta un formato de evaluación 360 el cual debe estar alineado a los objetivos de la institución. 
Revista Arbitrada Interdisciplinaria KOINONIA

Año IV. Vol IV. N${ }^{\circ}$. Edición Especial 2019

Hecho el depósito de Ley: FA2016000010

ISSN: 2542-3088

FUNDACIÓN KOINONIA (F.K). Santa Ana de Coro. Venezuela.

Pedro Eloy Lema Guiracocha; Juan Carlos Erazo Álvarez; Cecilia Ivonne Narváez Zurita

\section{Tabla 3}

\section{Formulario de evaluación de desempeño}

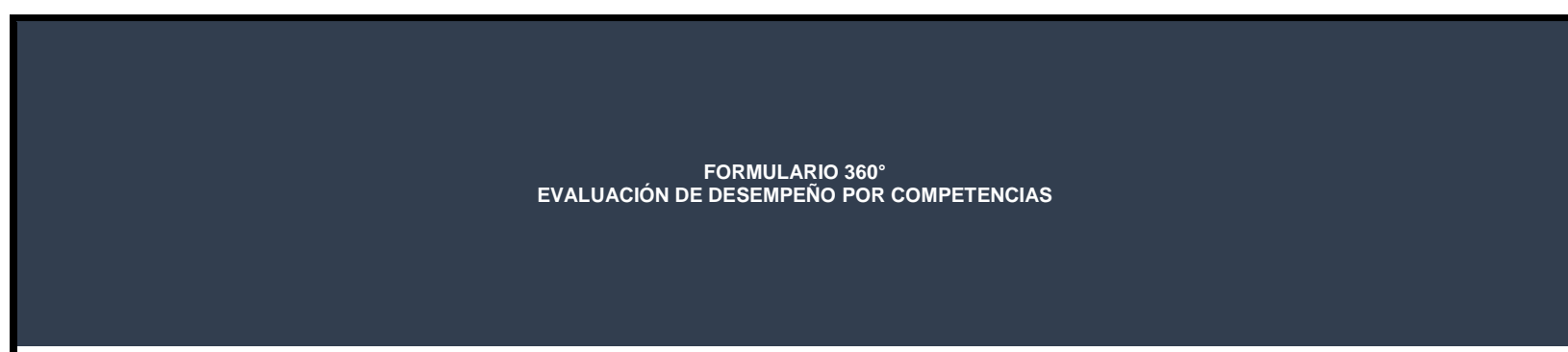

DATOS DEL EVALUADO:

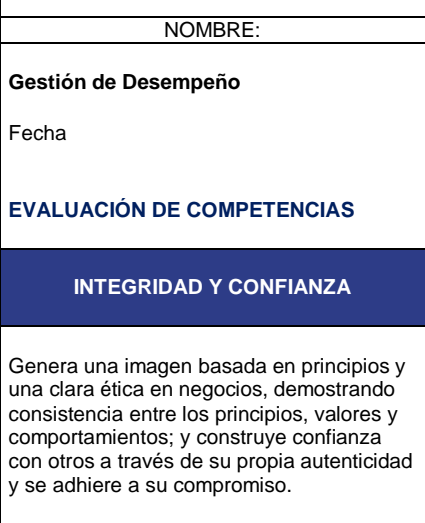

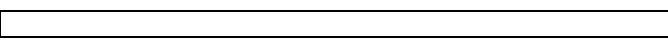

\begin{tabular}{|c|l|}
\hline CARGO: & \\
\hline
\end{tabular}

\begin{tabular}{l|}
\hline \multicolumn{1}{|c|}{ VOCACIÓN DE SERVICIO } \\
\hline
\end{tabular}

\begin{tabular}{|l|c|c|c|c|c|c|c|}
\multicolumn{2}{|c|}{ Nivel de Desarrollo de la Competencia } & \multicolumn{5}{c|}{ NIVEL DE OBSERVACION } \\
\cline { 2 - 7 } & $\mathbf{1}$ & $\mathbf{2}$ & $\mathbf{3}$ & $\mathbf{4}$ & $\mathbf{5}$ & $\mathbf{6}$ & $\mathbf{7}$ \\
\cline { 2 - 7 } & YO & JEF & COL & COL & COL & COL & COL \\
\hline $\begin{array}{l}\text { Trata a otros con dignidad y justicia. No exagera su propia capacidad } \\
\text { o de la organización. }\end{array}$ & & & & & & & \\
\hline $\begin{array}{l}\text { Protege conversaciones o información confidencial. Es honesto y } \\
\text { directo en su relación con otros. }\end{array}$ & & & & & & & \\
\hline $\begin{array}{l}\text { Hace promesas de valor y las cumple. Asume responsabilidad por su } \\
\text { comportamiento y sus errores. }\end{array}$ & & & & & & & \\
\hline $\begin{array}{l}\text { Presenta información verdadera a sus clientes y genera confianza } \\
\text { personal. }\end{array}$ & & & & & & & \\
\hline
\end{tabular}

\begin{tabular}{|c|c|}
\hline & \\
NIVEL DE OBSERVACION
\end{tabular}

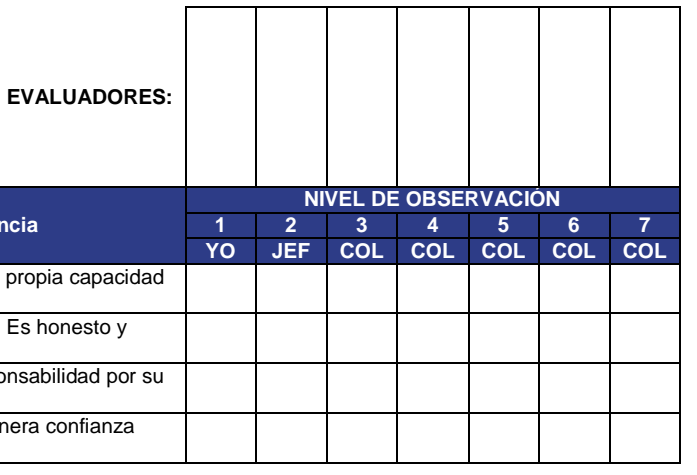

\section{Nivel de Desarrollo de la Competencia}

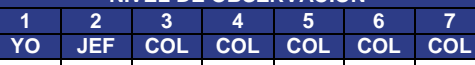

Se asegura de conocer adecuadamente los requerimientos de todos sus clientes, satisface las necesidades con agilidad y solvencia técnica y mejora continua de la organización.

Identifica proactivamente las necesidades del cliente, diagnostica

correctamente la necesidad y plantea soluciones adecuadas.

Actúa a partir de los pedidos de los clientes, ofreciendo respuestas estándar a sus demandas.

Da respuesta a las necesidades del cliente, muestra pocas

habilidades para brindar el servicio.

\section{VISIÓN ESTRATÉGICA DE NEGOCIO}

Capacidad de determinar eficazmente las metas y prioridades del negocio, definiendo las acciones, los plazos y los recursos

requeridos. Incluye la instrumentación de mecanismos de seguimiento, verificación y control; orientado al logro de resultados y fijando metas por encima de los estándares.

\section{Nivel de Desarrollo de la Competencia}

Define y comparte visiones de negocio. Orienta todo su accionar a la consecución de los Objetivos Estratégicos de la CACPE.

Es capaz de administrar simultáneamente diversas tareas complejas, orientadas a la Visión Estratégica del Negocio.

Establece objetivos y plazos para la realización de sus tareas, define prioridades para el cumplimiento de los objetivos de su área. Organiza sus tareas y administra adecuadamente los tiempos. Trabaja para cumplir los objetivos de su cargo.

\section{IMPACTO E INFLUENCIA}

Es la capacidad necesaria para generar credibilidad en los demás, sobre la base de los conocimientos técnicos de su

especialidad; con pleno convencimiento de que es capaz de influir con éxito en los demás. Ejerce liderazgo técnico.

\section{Nivel de Desarrollo de la Competencia}

Compromete a otros para realizar tareas adicionales, crea oportunidades y soluciona problemas.

Busca nuevas responsabilidades que generen impacto. Expresa su desacuerdo y presenta su posición en forma clara y segura.

Trabaja sin supervisión constante y no se amilana con los problemas; aparece seguro ante los demás.

En ocasiones se muestra poco seguro y necesita supervisión para la realización de su trabajo y el cumplimiento de los objetivos. 
Fuente: Elaboración propia

Finalmente se propone establecer un plan de incentivos hacia el personal de la cooperativa el cual se encuentra establecido de la siguiente manera:

\section{Tabla 4}

Plan de incentivos

\begin{tabular}{|c|c|c|c|}
\hline TIPO DE & OBJETIVO & CARACTERÍSTICA & BENEFICIOS \\
\hline 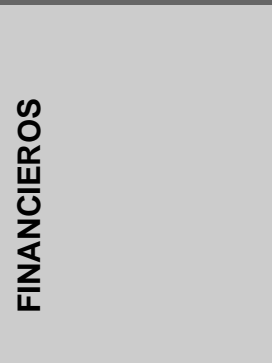 & $\begin{array}{l}\text { Es motivar a los } \\
\text { empleados para } \\
\text { mejorar el nivel de } \\
\text { productividad, así } \\
\text { como la consecución } \\
\text { de objetivos }\end{array}$ & $\begin{array}{l}\text { Incremento Sueldo } \\
\text { Comisiones } \\
\text { Bonos } \\
\text { Regalos(viajes) }\end{array}$ & $\begin{array}{l}\text { Reducción de rotación de } \\
\text { personal } \\
\text { Promover el trabajo en equipo } \\
\text { Aumentar la productividad } \\
\text { Personal mejor calificado } \\
\text { La no existencia de } \\
\text { favoritismos } \\
\text { Empleados comprometidos }\end{array}$ \\
\hline 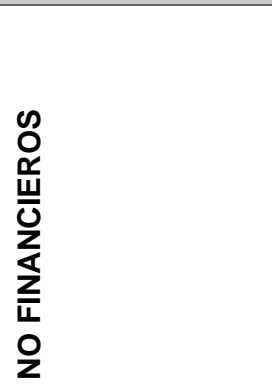 & & $\begin{array}{l}\text { Ascensos } \\
\text { Reconocimientos } \\
\text { Horarios flexibles } \\
\text { Días Libres } \\
\text { Planes médicos }\end{array}$ & \\
\hline
\end{tabular}

Fuente: Elaboración propia

Una gestión estratégica de los planes de compensación y beneficios se ha convertido hoy en día en un factor diferenciador con el propósito de atraer, retener y mantener a los mejores trabajadores, razón por la cual es importante establecer planes de compensación de las preferencias y de los momentos vitales de cada empleado, es decir deben ser más personalizables y entregarse con mayor frecuencia, razón por la cual se considera a la 
compensación como un factor diferenciador en un modelo de gestión de talento humano moderno.

Los resultados que se obtengan a través de la aplicación del modelo propuesto se convierten en acciones tendientes a mejorar el desempeño laboral de los empleados de la cooperativa, brindando así un servicio diferenciador hacia los socios y usuarios de la institución, además optimizando los tiempos y recursos de la cooperativa.

\section{CONCLUSIONES}

Es importante manifestar que se tiene que actualizar los diferentes manuales de procesos que mantiene la cooperativa y contar con un modelo de gestión de talento humano, que contribuya a mejorar el desempeño laboral, y su rendimiento, el cual permitirá alcanzar los objetivos estratégicos, su misión y visión, contribuyendo de esta forma el desarrollo de la Cooperativa de Ahorro y Crédito de la Pequeña Empresa Biblián Ltda.

La presente investigación pretende aportar con lineamientos que contribuyan a una mejor gestión del talento humano de la cooperativa, en donde se generen ventajas competitivas que me permita contar con un personal con las competencias y habilidades requeridas enfocadas en la propuesta de valor de la cooperativa que es el de brindar un servicio de calidad hacia los socios.

También es importante continuar capacitando al personal de la cooperativa, para mejorar la productividad y el desempeño laboral, el cual es traducido en la optimización de procesos, recursos que me permitan brindar un mejor servicio al socio.

El talento humano actualmente es la variable más competitiva de las organizaciones, ya que hoy en día las empresas están en la lucha por atraer, seleccionar y retener a los mejores talentos ya que estos permitirán generar mayores beneficios para las empresas. 


\section{REFERENCIAS CONSULTADAS}

1. Alles, M. (2006). Dirección Estratégica de Recursos Humanos Gestión por Competencias. Buenos Aires: Granica.

2. Aldana, J., \& Piña, J. (2017). Calidad del servicio prestado al cliente por los instructores de gimnasios. Revista Arbitrada Interdisciplinaria Koinonía, 2(3), 172 197. http://fundacionkoinonia.com.ve/ojs/index.php/revistakoinonia/article/view/59/46

3. Alvarado, N. M., \& Barba, A. M. (2016). Gestión del talento humano e innovación de la enseñanza y el aprendizaje. México: Palibrio.

4. Borrero, R. (2019). Procesos de gestión del talento humano en el sector educativo gerencial de Colombia. Revista Arbitrada Interdisciplinaria Koinonía, 4(7), 293-307. doi:http://dx.doi.org/10.35381/r.k.v4i7.206

5. Cerón Miranda, M. G., Erazo Álvarez, J. C., Ormaza Andrade, J. E., \& Narváez Zurita, C. I. (2019). La remuneración variable como eje estratégico de la gestión de Talento Humano en la Unidad de Negocio Hidropaute. Visionario Digital, 20.

6. Chiavenato, I. (2011). Administración de Recursos Humanos. Santafé de Bogotá, Colombia: McGraw-Hill, Novena Edición.

7. Cordero Cordero, J. J., Narváez Zurita, C. I., \& Erazo Álvarez, J. C. (2019). La evaluación por competencias: una herramienta para determinar la productividad del talento humano. 593 Digital Publisher CEIT, 72-73.

8. Dessler, G. (2009). Administración de Recursos Humanos. México: Pearson.

9. García Solarte, M. (2009). Los macro-procesos: un nuevo enfoque en el estudio de la Gestión Humana. Pensamiento \& Gestión, (27), 162-200.

10. Ramos, A. (2014). La gestión del talento humano y el desempeño laboral en la Cooperativa de Ahorro y Crédito Educadores de Pastaza. Puyo - Pastaza: Universidad regional Autónoma de los Andes "UNIANDES".

11. Rodríguez, J. (2012). El modelo de gestión de recursos humanos. Cuarta edición. Barcelona: UOC.

12. Superintendencia de Economía Popular y Solidaria, S. (2018). Una mirada al desarrollo de la Economía Popular y Solidaria. Quito: Boletín SEPS 11. 
13. Tejada, A. (2003). Los Modelos actuales de Gestión en las organizaciones. Gestión del Talento, Gestión del Conocimiento y Gestión por Competencias. Psicología desde el Caribe, 12, 115-133.

14. Vallejo L. M. (2016). Gestión del Talento Humano. Ecuador: Caracola Editores. Recuperado desde: http://cimogsys.espoch.edu.ec/direccionpublicaciones/public/pdf/18/gesti\%C3\%B3n\%20del\%20talento\%20humano. Pdf

\section{REFERENCES CONSULTED}

1. Alles, M. (2006). Strategic Human Resources Management Skills Management. Buenos Aires: Granica.

2. Aldana, J., \& Piña, J. (2017). Quality of service provided to the client by gym instructors. Interdisciplinary Arbitrated Review Koinonía, 2 (3), 172-197. Recovered from http://fundacionkoinonia.com.ve/ojs/index.php/revistakoinonia/article/view/59/46

3. Alvarado, N. M., \& Barba, A. M. (2016). Human talent management and innovation in teaching and learning. Mexico: Palibrio.

4. Borrero, R. (2019). Human talent management processes in the management education sector of Colombia. Interdisciplinary Arbitrated Review Koinonía, 4 (7), 293-307. doi: http: //dx.doi.org/10.35381/r.k.v4i7.206

5. Cerón Miranda, M. G., Erazo Álvarez, J. C., Ormaza Andrade, J. E., \& Narváez Zurita, C. I. (2019). Variable remuneration as a strategic axis of Human Talent management in the Hidropaute Business Unit. Digital Visionary, 20.

6. Chiavenato, I. (2011). Human resources management. Santafé de Bogotá, Colombia: McGraw-Hill, Ninth Edition.

7. Cordero Cordero, J. J., Narváez Zurita, C. I., \& Erazo Álvarez, J. C. (2019). Competency assessment: a tool to determine the productivity of human talent. 593 Digital Publisher CEIT, 72-73.

8. Dessler, G. (2009). Human resources management. Mexico: Pearson.

9. García Solarte, M. (2009). The macro-processes: a new approach in the study of Human Management. Thought \& Management, (27), 162-200. 
10.Ramos, A. (2014). Human talent management and work performance in the Pastaza Educators Savings and Credit Cooperative. Puyo - Pastaza: Regional University of the Andes "UNIANDES".

11. Rodríguez, J. (2012). The human resources management model. Fourth edition. Barcelona: UOC.

12. Superintendencia de Economía Popular y Solidaria, S. (2018). A look at the development of the Popular and Solidarity Economy. Quito: SEPS Bulletin 11.

13. Tejada, A. (2003). Current Management Models in organizations. Talent Management, Knowledge Management and Skills Management. Psychology from the Caribbean, 12, 115-133.

14. Vallejo L. M. (2016). Human Talent Management. Ecuador: Caracola Editores. Recovered from: http://cimogsys.espoch.edu.ec/direccionpublicaciones/public/pdf/18/gesti\%C3\%B3n\%20del\%20talento\%20humano. PDF 